\title{
Improvement of Adaptive Smart Concentric Circular Antenna Array Based Hybrid PSOGSA Optimizer
}

\author{
Ahmed Magdy \\ Department of Electrical \\ Engineering \\ Minia University \\ Minia, Egypt
}

\author{
Osama M. EL-Ghandour \\ Department of Elect., Commu., and \\ Comp. Engineering Helwan \\ university \\ Cairo, Egypt
}

\author{
Hesham F. A. Hamed \\ Department of Electrical \\ Engineering \\ Minia University \\ Minia, Egypt
}

\begin{abstract}
Unlike all recent research which used Concentric Circular Antenna Array (CCAA) based on one beam-former for each single main beam, this research presents a technique to adapt smart CCAA by using only single beam-former for multi main beams based on hybrid PSOGSA. Hybrid PSOGSA is a combining technique between Particle Swarm Optimization and Gravitational Search Algorithm which is applied in the feeding of the smart CCAA to enhance its performance. Phase excitation of the array with a large number of elements is suggested for different scenarios based on hybrid PSOGSA and other algorithms such as PSO and GSA in High Altitude Platform (HAP) application. Simulation results proved that hybrid PSOGSA achieves better performance than other optimizers for excitation of the smart CCAA in all scenarios for different parameters like normalized array factor, fitness values, convergence rate, and directivity.
\end{abstract}

Keywords-Smart antenna; CCAA; PSOGSA; HAP; and Beamforming

\section{INTRODUCTION}

Smart antenna was one of the main parts in various communication systems [1-3]. Circular Antenna Array (CAA) $[4,5]$ was considered recently in the latest research because of its ability to steer the radiation pattern more than linear antenna arrays and overcoming the broadside problem. CCAA implements different rings with different radius that are used in most wireless applications such as High Altitude Platform (HAP) and others [6-10].

Particle Swarm Optimization (PSO) in [11] was used in a MIMO system in [12]. Cooperative communication based on one and multi relay was proposed by using PSO in $[13,14]$. The suggested Gravitational Search Algorithm (GSA) in [15] was used for Direction of Arrival estimation in smart antenna systems and achieved better results than MUSIC and PSO in [16]. Synthesis CCAA was studied by GSA and modified PSO in [17]. GSA was used to control reconfigurable dual-beam for the CCAA and steer null toward SNOI using Linear Constraint Minimum Variance Assisted by different optimization techniques, finally GSA gave the best performance in $[18,19]$.

Recently, Hybrid Particle Swarm Optimization with Gravitational Search Algorithm (Hybrid PSOGSA) technique was considered as a new optimization technique that showed better performance than standard PSO and GSA in terms of computational speed and fitness values [20,21]. In [22], an algorithm based on collective animal behaviour (CAB) is used for finding the best optimal excitation weights for linear antenna arrays, but in [4,5], hybrid PSOGSA utilizing UCA achieved better results than ULA in terms of studying the directed power in terms of normalized array factor toward the intended direction in addition to direct null to signal Not of interest (SNOI) for different scenarios.

In this paper, unlike all previous research which was based on multi beam-former for multi beams like [17-19,22,23] or small number of elements like [4-5], a novel algorithm using only one beam-former for multi beams that is based on the hybrid PSOGSA technique is utilized for optimal beamforming using CCAA which implement a greater number of elements up to 121 elements. The main aim based on controlling the complex weights in order to maximize and minimize the beam of the radiation pattern towards the desired user (SOI) or not desired (SNOI) respectively. The paper is organized as follows. In Section II, the optimization model and problem formulation for adaptive beam-forming is suggested. In Section III, hybrid PSOGSA algorithm is proposed. Simulation results and discussions for beam-forming are discussed in Section IV. Finally, Section V explains the conclusions.

\section{OPTIMIZATION PROBLEM MODEL}

CCAA is suggested for Smart antenna, as shown in Figure 1. CCAA with $\mathrm{N}$ elements are distributed along $\mathrm{M}$ circles of radius $\mathrm{r}_{\mathrm{m}}$ and each circle has $\mathrm{N}_{\mathrm{m}}$ elements, The Array factor for CCAA can be defined from[7] as shown:

$$
\begin{aligned}
& \operatorname{AF}(\theta)=\sum_{m=1}^{M} \sum_{n=1}^{N_{m}}\left[\cos \left(\beta * r_{m} * \cos \left(\theta-\operatorname{pos}_{m n}\right)-\alpha_{m n}\right)+\right. \\
& \left.j \sin \left(\beta * r_{m} * \cos \left(\theta-\operatorname{pos}_{m n}\right)-\alpha_{m n}\right)\right]
\end{aligned}
$$

Where $\lambda$ is the wavelength, the angle between adjacent elements is $\theta_{0}, \theta$ is the azimuth angle, $d=0.5 \lambda$ is the space between two adjacent elements, $\beta$ is the phase shift constant, $\alpha_{m n}$ represents the complex excitation phase of the mn-th element and $\operatorname{pos}_{\mathrm{mn}}$ is the angular position of the mn-th element. The studying for radiation pattern shows the phase excitation for elements in smart array must minimize the radiation power intensity at certain directions (SNOI) and maximize the main-lobes to other directions (SOI). Our cost function is modified than cost function in [13] where we maximized the total output power toward the desired signal at $\theta_{\mathrm{i}}$ and minimized the total output power in the direction of the interfering signals at $\theta_{j}$ and this done by minimizing the fitness function in (2). 
fitness function $=-\sum_{\mathrm{i}=1}^{\mathrm{k}} \mathrm{a}_{\mathrm{i}} \mathrm{AF}\left(\theta_{\mathrm{i}}\right)+\sum_{\mathrm{i}=1}^{\mathrm{L}} \mathrm{b}_{\mathrm{i}} \mathrm{AF}\left(\theta_{\mathrm{i}}\right)$

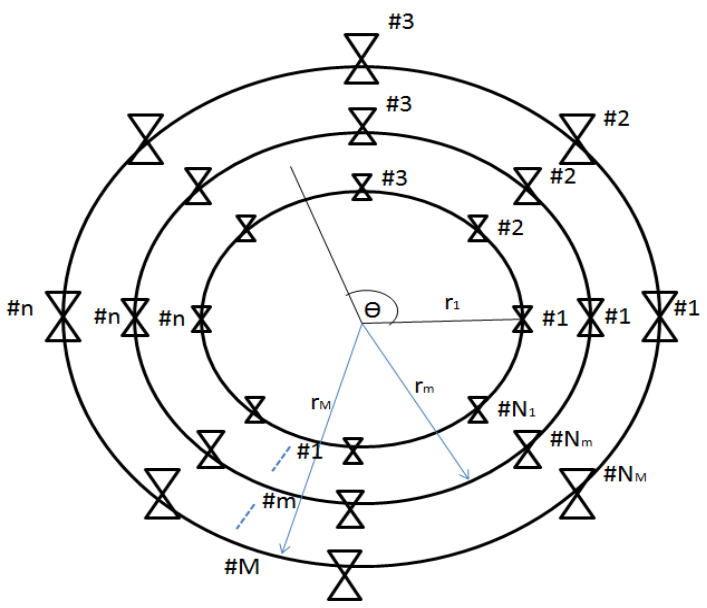

(2)

Fig. 1. Structure of the smart CCAA

In this paper, CCAA uses number of elements $\mathrm{N}=121$ for huge application like High altitude Platform (HAP) as shown in $[6,23]$. When PSOGSA is compared with other previous algorithms like PSO and GSA according to many factors such as normalized array factor using(1), directivity using (3) as shown in [7] and computational speed with convergence rate for normalized fitness values using (2).

$D=4 \pi / \int_{0}^{2 \pi} \int_{0}^{\pi}\left|A F_{n}\right|^{2} \sin \phi d \phi d \theta$

Where $\phi$ elevation angle which is used 900 in our work and $\theta$ is azimuth angle.

\section{HYBRID PSOGSA OPTIMIZER}

In this paper, PSO and GSA were combined with a parallel hybridized method not series method and for this reason the hybridization method was called co-evolutionary. On the other hand, the low level combines the functionality of both algorithms. It is heterogeneous because there are two different algorithms that are involved in producing final results. The main idea of hybrid PSOGSA is a combination between capability of local search by GSA and social think the ability by PSO. Velocity is updated as follows from [20,21]:

$\mathrm{v}_{\mathrm{i}}(\mathrm{t}+1)=$ part $1+$ part 2

Where part 1 of the GSA [15] : $\mathrm{w} * \mathrm{v}_{\mathrm{i}}(\mathrm{t})+\mathrm{C} 1 *$ rand $*$ $\mathrm{a}_{\mathrm{i}}(\mathrm{t})$ and part 2 of the PSO [11] : C2 $*$ rand $*\left(\right.$ gbest $\left.-\mathrm{x}_{\mathrm{i}}(\mathrm{t})\right)$ finally, velocity can be updating by

$$
\mathrm{v}_{\mathrm{i}}(\mathrm{t}+1)=\mathrm{w} * \mathrm{v}_{\mathrm{i}}(\mathrm{t})+\mathrm{C} 1 * \operatorname{rand} * \mathrm{a}_{\mathrm{i}}(\mathrm{t})+\mathrm{C} 2 * \operatorname{rand} *
$$

(gbest $\left.-\mathrm{x}_{\mathrm{i}}(\mathrm{t})\right)$

Every iteration, the positions of agents are updated as follows:

$\mathrm{x}_{\mathrm{i}}(\mathrm{t}+1)=\mathrm{x}_{\mathrm{i}}(\mathrm{t})+\mathrm{v}_{\mathrm{i}}(\mathrm{t}+1)$

Where gbest is global best position from PSO, $w$ is a weighting factor, rand is a random number, C1 and $\mathrm{C} 2$ are constant, $\mathrm{v}_{\mathrm{i}}(\mathrm{t})$ is the velocity of particlei at iteration $\mathrm{t}$ and $\mathrm{a}_{\mathrm{i}}(\mathrm{t})$ is the acceleration of agent $\mathrm{i}$ at iteration $\mathrm{t}$. all these parameters are taken from PSO and GSA algorithm [20, 21]. Finally Hybrid PSOGSA procedures are shown in Figure 2.

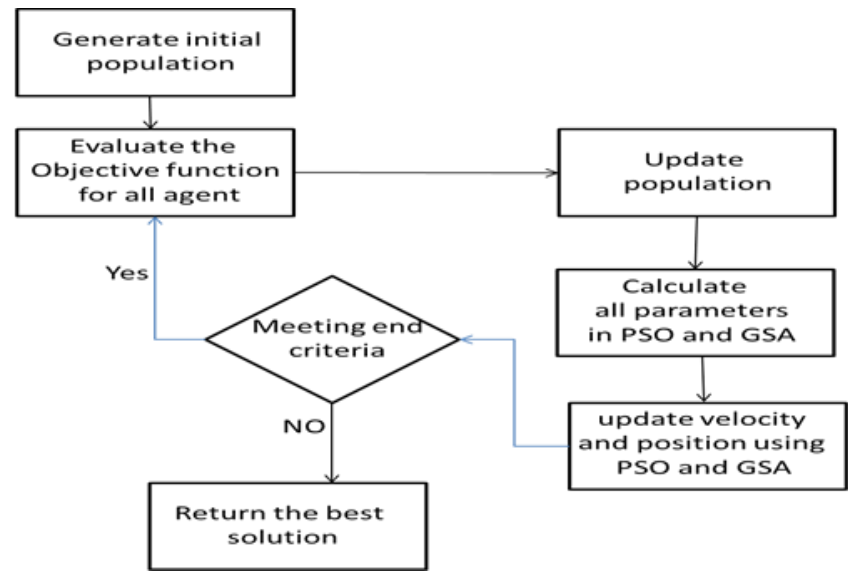

Fig. 2. Flow chart of procedures for hybrid PSOGSA optimizer

As shown in Figure 2, the first step is responsible for all agents are randomly initialized. Each agent is considered as a candidate solution. As can be seen in the second step, after initialization, evaluate the fitness function based on equation (2). At the third and fourth step, gravitational force, gravitational constant, resultant forces among agents are calculated, and the accelerations of particles are defined from $[20,21]$. In each iteration, the best solution so far (gbest) must be updated. After calculating the accelerations and with updating the best solution so far, the velocities and the positions of all agents can be updated from [20,21] at the fifth step. Finally, after the agents are updated. The process of updating velocities and positions will be stopped by meeting an end criterion.

\section{SimUlation AND Discussion RESUltS}
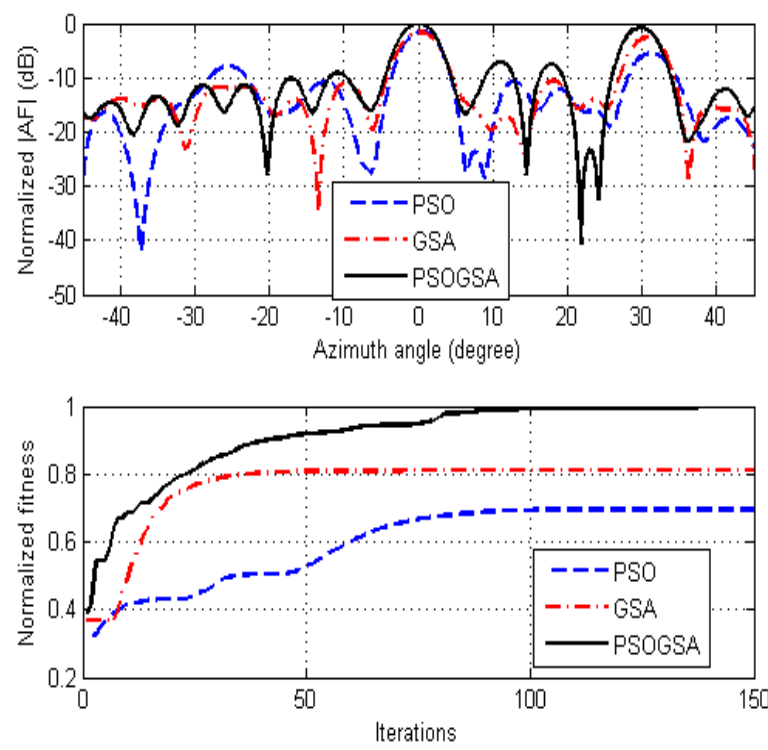

Fig. 3. The first scenario for normalized array factor versus azimuth angle and fitness functions versus iteration using Hybrid PSOGSA, PSO and GSA based on CCAA topology 
MATLAB package is used for evaluating the above analysis. In this section, ability of adaptive beam-forming based on hybrid PSOGSA technique using CCAA is studied. All algorithms are employed with a population size of 30 and 150 iterations which represent a small number of iterations. Figures 3,4,5,6, and 7 show comparison of PSOGSA with GSA and PSO using CCAA topology for many scenarios in terms of radiation pattern, convergence curve and computation time, in order to compute directivity. The work implemented 121 elements in all scenarios for HAP application.
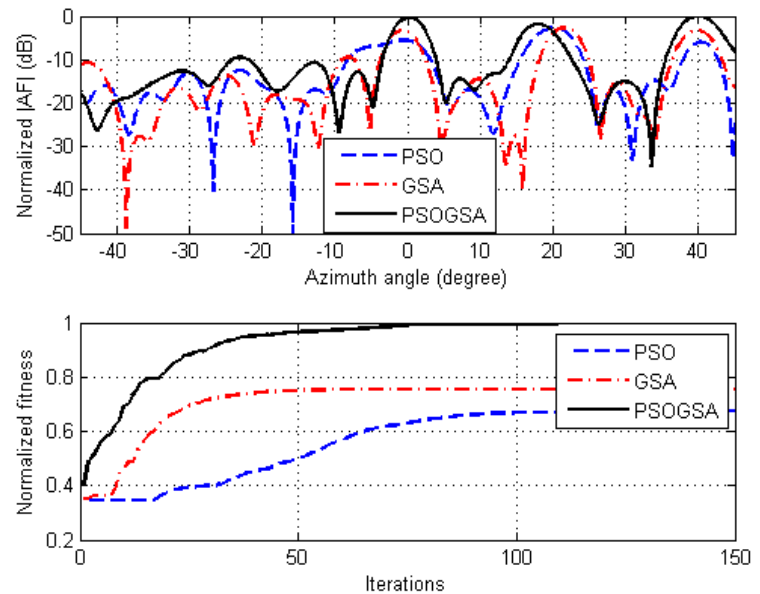

Fig. 4. The second scenario for normalized array factor versus azimuth angle and fitness functions versus iteration using Hybrid PSOGSA, PSO and GSA based on CCAA topology
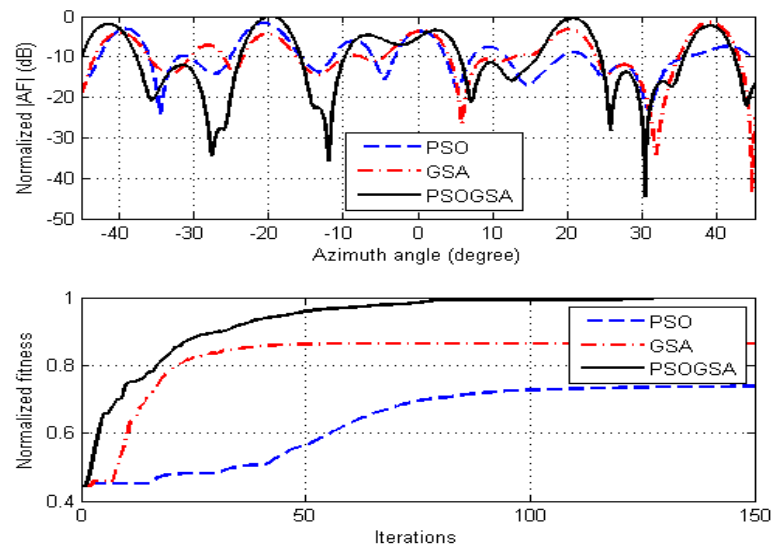

Fig. 5. The third scenario for normalized array factor versus azimuth angle and fitness functions versus iteration using Hybrid PSOGSA, PSO and GSA based on CCAA topology
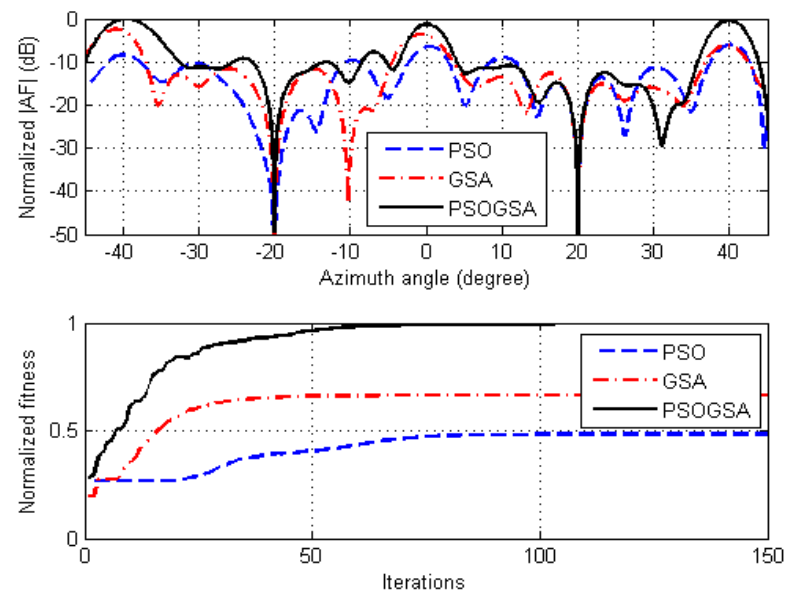

Fig. 6. The fourth scenario for normalized array factor versus azimuth angle and fitness functions versus iteration using Hybrid PSOGSA, PSO and GSA based on CCAA topology
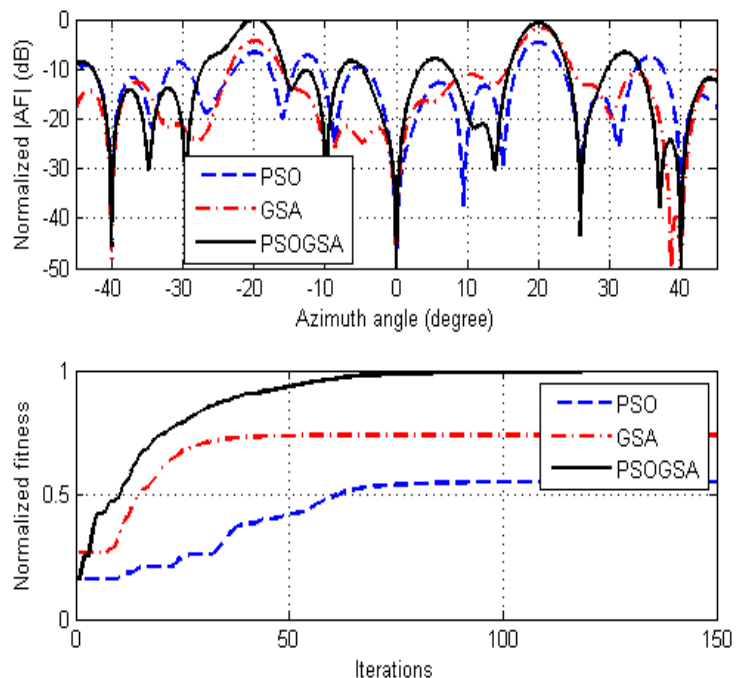

Fig. 7. The fifth scenario for normalized array factor versus azimuth angle and fitness functions versus iteration using Hybrid PSOGSA, PSO and GSA based on CCAA topology

All scenarios can be discussed in Tables 1 and 2. Table 1 indicates that hybrid PSOGSA in general outperforms PSO and GSA. The hybrid PSOGSA is better than PSO and GSA in all scenarios by range from -2.8 to -6.65 and from -1.4 to -3.85 respectively in terms of averages normalized array factor in $\mathrm{dB}$. 
TABLE I. THE CORRESPONDING AVERAGE NORMALIZED $\mid$ AF $\mid$ VALUES AT THE SOI AND SNOI DIRECTIONS FOR DIFFERENT SCENARIOS BASED ON CCAA TOPOLOGY

\begin{tabular}{|c|c|c|c|c|c|c|c|c|}
\hline \multirow{3}{*}{ 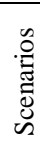 } & \multirow{3}{*}{ 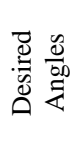 } & \multirow{3}{*}{ 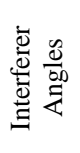 } & \multicolumn{6}{|c|}{ Average Normalized array factor $(\mathrm{dB})$} \\
\hline & & & \multicolumn{2}{|c|}{$P S O$} & \multicolumn{2}{|c|}{ GSA } & \multicolumn{2}{|c|}{ PSOGSA } \\
\hline & & & Major & Null & Major & Null & Major & null \\
\hline 1 & [0 30] & ---- & -3.8 & $\begin{array}{ll}----- \\
\end{array}$ & -2.2 & $\begin{array}{ll}----- \\
\end{array}$ & -0.35 & $\begin{array}{c}----- \\
\end{array}$ \\
\hline 2 & $\begin{array}{c}{\left[\begin{array}{ll}0 & 20 \\
40\end{array}\right]}\end{array}$ & --- & -4.7 & $\begin{array}{ll}----- \\
\end{array}$ & -3.5 & $\begin{array}{c}----- \\
\end{array}$ & -1.2 & ----- \\
\hline 3 & $\begin{array}{c}{[-40-} \\
200 \\
2040]\end{array}$ & --- & -5 & ----- & -3.6 & ----- & -2.2 & 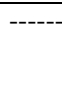 \\
\hline 4 & $\begin{array}{c}{[-400} \\
40]\end{array}$ & $\begin{array}{c}{[-20} \\
20]\end{array}$ & -7 & $>-40$ & -4.2 & $>-40$ & -0.35 & $>-40$ \\
\hline 5 & $\begin{array}{c}{[-20} \\
20]\end{array}$ & 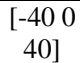 & -5.5 & $>-40$ & -3 & $>-40$ & -0.35 & $>-40$ \\
\hline
\end{tabular}

On the other hand, from table 2, simulation results have shown tremendous improvement over the previous work done using standard PSO and GSA in terms of directivity and convergence speed for normalized fitness values. PSOGSA is better than PSO and GSA by $25 \%-50 \%$ and $15 \%-30 \%$, respectively in terms of convergence percentage at the end of iteration. It was shown that PSOGSA achieved improvement over GSA and PSO by 1\%-70\% nearly in terms of directivity. The only disadvantage in PSOGSA is computational time. Still, all algorithms have the ability to produce null toward SNOI by more than $-40 \mathrm{~dB}$ at scenario \#4 and \#5.

TABLE II. THE CORRESPONDING NORMALIZED CONVERGENCE PeRCENTAGE AT THE END OF ITERATIONS, THE DiRECTIVITY VALUE AT THE MAX SOI DIRECTIONS AND COMPUTATIONAL TIME FOR DIFFERENT SCENARIOS BASED ON CCAA TOPOLOGY

\begin{tabular}{|c|c|c|c|c|c|c|c|c|c|}
\hline \multirow{2}{*}{ 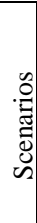 } & \multicolumn{3}{|c|}{$\begin{array}{c}\text { Normalized } \\
\text { Convergence } \\
\text { percentage } \\
\text { (nearly) \% }\end{array}$} & \multicolumn{3}{|c|}{$\begin{array}{c}\text { Directivity } \\
\text { (dimensionless) }\end{array}$} & \multicolumn{3}{|c|}{$\begin{array}{l}\text { computational time in } \\
\text { sec using Lenovo core } \\
\text { i } 7 \text { 4702MQ RAM } 8 \text { G }\end{array}$} \\
\hline & $\mathrm{PSO}$ & $G S A$ & $\begin{array}{l}P S O \\
G S A\end{array}$ & $P S O$ & $G S A$ & $\begin{array}{l}P S O \\
G S A\end{array}$ & $\mathrm{PSO}$ & $G S A$ & $\begin{array}{l}P S O \\
G S A\end{array}$ \\
\hline 1 & 70 & 80 & 100 & 15.63 & 16.4 & 17.4 & 1 & 0.5 & 1.5 \\
\hline 2 & 65 & 75 & 100 & 9.680 & 11.7 & 12.5 & 1 & 0.5 & 1.3 \\
\hline 3 & 75 & 85 & 100 & 7.975 & 9.40 & 9.52 & 1.7 & 0.9 & 2 \\
\hline 4 & 50 & 70 & 100 & 8.008 & 10.7 & 10.8 & 1.7 & $\begin{array}{l}0.9 \\
\end{array}$ & 2.1 \\
\hline 5 & 55 & 75 & 100 & 5.665 & 14.4 & 14.9 & 1.6 & 0.8 & 1.9 \\
\hline
\end{tabular}

\section{CONCLUSION}

In this paper, a hybrid PSOGSA technique is implemented only on one beam-former for multi-beams smart antenna systems based on CCAA to improve the capability of smart beam-former in wireless communication systems. It is clear from our optimization model using CCAA, simulation results have shown tremendous improvement over the previous work done using standard PSO and GSA in terms of normalized array factor by range from -2.8 to -6.65 and from -1.4 to -3.85 respectively, directivity by range from $1 \%$ to $70 \%$, and convergence speed for normalized fitness values by range from $25 \%$ to $50 \% \mathrm{~dB}$ and from $15 \%$ to $30 \%$, respectively. However, simulation results and discussions proved the ability of our technique to deal with a big set of simultaneously incident and interfere angles. It was found that hybrid PSOGSA based on CCAA is the best powerful optimizer for smart beam-forming applications and better than GSA and PSO on average for several scenarios.

\section{REFERENCES}

[1] Ahmed Magdy, S. Sayed, K.R.Mahmoud , "Modified Cooperative Access with Relay's Data Protocol using smart antenna," Journal of Engineering Sciences, Assiut University, Faculty of Engineering, Vol. 41, No. 6, November, 2013.

[1] Ahmed Magdy, K. R. Mahmoud, S. Sayed, and I. I. Ibrahim, "Modified Cooperative Access with Relay's Data (MCARD)," Production and hosting by Elsevier and sciencedirect B.V. on behalf of Faculty of Engineering, Alexandria University, Volume 53, Issue 1, Pages 33-40, March 2014.

[2] GhouseBasha, T.S.; Aloysius, G.; Rajakumar, B.R.; Giri Prasad, M.N.; Sridevi, P.V., "A constructive smart antenna beam-forming technique with spatial diversity," in Microwaves, Antennas \& Propagation, IET, vol.6, no.7, pp.773-780, May 162012

[3] Ahmed Magdy, Osama M. El-Ghandour, and Hesham F. A. Hamed, "Performance Enhancement for Adaptive Beam-Forming Application Based Hybrid PSOGSA Algorithm," Journal of Electromagnetic Analysis and Application, 7, 126-133, 2015.

[4] S. S. S. Chakravarthy Vedula, S. R. Chowdary Paladuga, and M. RAO PRITHVI, "SYNTHESIS OF CIRCUlAR ARRAY ANTENNA FOR Sidelobe Level and APERTURe Size Control Using Flower POLLINATION ALGORITHM,"INTERNATIONAL JOURNAL OF ANTENNAS AND PROPAGATION, VOL. 2015, PP. 1-9, 2015

[5] Sultan Aljahdali, " Enhancing the Capacity of Stratospheric Cellular Networks Using Adaptive ArrayTechniques," I. J. Computer Network and Information Security, 2013, 6, 1-10, Published Online May 2013

[6] D. Manda1, V.P.Roy, A. Chattrejee, and A. K. Bhattacharjee," Side Lobe Reduction of Concentric Thinned Antenna Array Using Evolutionary Algorithms," IOSR Journal of Electrical and Electronics Engineering, Volume 9, Issue 3 Ver. III, PP 43-49 (May - Jun. 2014).

[7] S. Pal, A. Basak, S. Das, A. Abraham and I. Zelinka, "Concentric Circular Antenna Array synthesis using a differential Invasive Weed Optimization algorithm," Soft Computing and Pattern Recognition (SoCPaR), 2010 International Conference of, Paris, 2010, pp. 395-400.

[8] K. Rajesh Kumar, S. Aruna, and K. SrinivasaNaik," Side Lobe Reduction of a Concentric Circular Antenna Array using Genetic Algorithm (GA) and Particle Swarm Optimization (PSO)," International Journal of Engineering and Innovative Technology (IJEIT), Volume 4, Issue 5, November 2014

[9] D. Mandal, R. Kar and S. P. Ghoshal, "Thinned concentric circular array antenna synthesis using Particle Swarm Optimization with Constriction Factor and Inertia Weight Approach," Recent Trends in Information Systems (ReTIS), 2011 International Conference on, Kolkata, 2011, pp. 77-81.

[10] Kennedy, J. and R. Eberhart, "Particle swarm optimization," IEEE International Conference on Neural Networks, Vol. 4, 1942-1948, Perth, Australia, 1995.

[11] Ho, M.-H.; Chiu, C.-C.; Liao, S.-H., "Optimisation of channel capacity for multiple-input multiple-output smart antenna using a particle swarm optimiser," in Communications, IET, vol.6, no.16, pp.2645-2653, November 62012.

[12] Ahmed Magdy, K. R. Mahmoud, and S. Sayed, "Cooperative Communications Based Smart Antenna Systems Using PSO Algorithm," PIERS Proceedings, Stockholm, Sweden, Aug. 12-15, pp.600-615, 2013.

[13] Ahmed Magdy, K. R. Mahmoud, S. Sayed, and I. I. Ibrahim, "Smart Circular Antenna Array Based Cooperative Communications using Optimization Technique," Journal of Engineering Sciences, Assiut University, Faculty of Engineering, Vol. 41, No. 5, September, 2013. 
[14] Rashedi, E., H. Nezamabadi-Pour, and S. Saryazdi, "GSA: A gravitational search algorithm," Information Sciences, Vol. 179, No. 13, 2232-2248, 2009

[15] Ahmed Magdy, K. R. Mahmoud, S. G. Abdel-Gawad, and I. I. Ibrahim, "Direction of Arrival Estimation Based on Maximum Likelihood Criteria Using Gravitational Search Algorithm," PIERS Proceedings, Taipei, March 25-28,pp. 1162-1167, 2013.

[16] Chatterjee, A., G. K. Mahanti, and N. N. Pathak, "Comparative performance of gravitational search algorithm and modified particle swarm optimization algorithm for synthesis of thinned scanned concentric ring array antenna," Progress In Electromagnetics Research B, Vol. 25, 331-348, 2010.

[17] Chatterjee, A., G. K. Mahanti, and P. R. S. Mahapatra, "Design of fully digital controlled reconfigurable dual-beam concentric ring array antenna using gravitational search algorithm,"Progress In Electromagnetics Research C, Vol. 18, 59-72, 2011.

[18] SoodabehDarzi, TiongSiehKiong, Mohammad Tariqul Islam, Mahamod Ismail, SalehinKibria, and Balasem Salem, "Null Steering of Adaptive Beamforming Using Linear Constraint Minimum Variance Assisted by Particle Swarm Optimization, Dynamic Mutated Artificial Immune
System, and Gravitational Search Algorithm," The Scientific World Journal, vol. 2014, Article ID 724639, 10 pages, 2014.

[19] SeyedaliMirjalili, and SitiZaitonMohdHashim, "A New Hybrid PSOGSA Algorithm for Function Optimization," International Conference on Computer and Information Application, ICCIA, IEEE 2010.

[20] BinjieGu and Feng Pan, "MODIFIED GRAVITATIONAL SEARCH ALGORITHM WITH PARTICLE MEMORY ABILITY AND ITS APPLICATION," International Journal of Innovative Computing, Information and Control, Volume 9, Number 11, November 2013.

[21] Gopi Ram, Durbadal Mandal, RajibKar, and Sakti Prasad Ghoshal, " Optimized Hyper Beamforming of Linear Antenna Arrays Using Collective Animal Behaviour," The ScientificWorld Journal, Article ID 982017, 13 pages, Volume 2013.

[22] Yasser Albagory, "Flat-Top Ring-Shaped Cell Design for High-Altitude Platform Communications," I. J. Computer Network and Information Security, 2013, 7, 51-57, Published Online June 2013.

[23] C.A. Balanis, Antenna theory: Analysis and design,3rd ed., Wiley, New York, 2005. 